\section{Is minimal residual disease a convincing tool to determine the treatment duration of immune checkpoint inhibitors?}

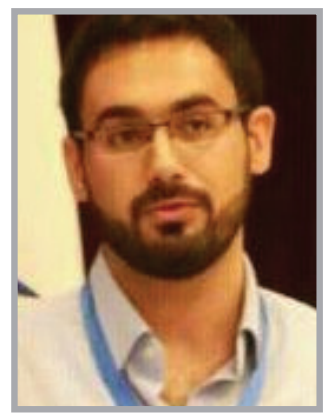

\author{
"The use of circulating tumor cells to monitor \\ minimal residual disease as a surrogate for efficacy \\ and duration of treatment remains quite \\ speculative and is prone to major technical \\ problems."
}

Elie El Rassy', Tarek Assi*,1 \& Joseph Kattan ${ }^{1}$

First draft submitted: 14 October 2016; Accepted for publication: 10 November 2016; Published online: 25 November 2016

The treatment armamentarium of cancer has evolved tremendously in the last decades with the concept of targeted drugs [1]. Immune checkpoint inhibitors (ICI) is a targeted treatment that deactivates the tumor-induced inhibition of the immune system [2]. Unfortunately, ICI are administered continuously until disease progression or occurrence of limiting toxicity in view of the absence of data that specifies the length of ICI administration. The toxicity profile of ICI depends on the type of inhibitor used. The toxicities of CTLA-4 inhibitors are expected as early as 3 weeks after treatment initiation while that of the PD-1/PDL-1 inhibitors occur later starting 6 weeks after the first dose. The number and severity of these toxicities seem to escalate with time [3,4]. Effectively, the earliest adverse events are limited to pruritus and rash $[3,4]$. Thereafter, more serious toxicities follow including colitis, endocrinopathies, pneumonitis and renal failures. The available data do not describe a cumulative toxicity with the prolonged administration of ICI [3,4]. However, one should keep in mind the potential occurrence of cytokine release syndrome and severe financial toxicities with the prolonged treatment durations. In this editorial, we highlight the unanswered questions concerning the duration, toxicity and gauging efficacy of ICI based on the limited publications available.

One potential approach for the follow-up of patients receiving ICI could be extrapolated from the concept of minimal residual disease (MRD) used in leukemic patients. It represents a real-time estimation of the patient's tumor burden thus estimates the count of tumor-related markers defined according to a cut-off point [5]. Patients with solid tumors treated with ICI could potentially benefit from this concept. It has been first applied in the 90s in patients with advanced melanoma and then extrapolated to other solid tumors [6]. Effectively, it has been studied in breast and colon cancers

\section{KEYWORDS}

- circulating tumor cells $\bullet$ immune checkpoint inhibitors $\bullet$ minimal residual disease

\section{"Immune checkpoint inhibitors is a targeted treatment that deactivates the tumor-induced inhibition of the immune system.”}


"Immune checkpoint inhibitors is a targeted treatment that deactivates the tumor-induced inhibition of the immune system." where it was demonstrated to be a prognostic factor for recurrence and a determinant factor for more aggressive treatment [7-9].

The available data concerning this issue are limited with ICI. The first study that evaluated the role of MRD in patients receiving ICI was conducted in metastatic non-small-cell lung cancer patients. The MRD was determined by the levels of circulating tumor cells (CTC) measured at baseline, at 3 and 6 months [10]. At 6 months, patients with persistent PD-L1-positive CTC had progressive disease while those with negative PD-L1 benefited from an objective response [10]. These findings are in line with the survival curves comparing ICI of Phase III trials that tend to separate after 6 months of treatment. Therefore, the concept of CTC might apply in patients receiving ICI but yet requires a more robust validation. Our review of the pivotal ICI trials reported a toxicity-related dropout rate of 3-8\% [11,12]. We believe that reporting on the response duration and survival of these patients would determine the tumors' and patients' characteristics that might potentially benefit from a limited duration of ICI administration.

The recently reported results by Reck $e t$ al. of the KEYNOTE-024 have led to the approval of pembrolizumab in the first line setting of nonsmall-cell lung cancer. In this study, we observe a trend to limit the duration of ICI administration in the first line setting by predetermining 35 cycles of ICI in the study arm [13]. Such study design does not preclude patients from benefiting the most from ICI treatment. Effectively, the withdrawal of ICI after a predetermined number of cycles is not associated with an increased risk of acquired resistances such as that described with cytotoxic chemotherapy. We read with interest the observational study by Lipson $e t$ al. that describes the persistence of objective response in patients receiving ICI even after treatment discontinuation. In this case series, rechallenging the tumor with the same ICI even after a long honeymoon period demonstrated a greater response and lesser adverse events [14]. The exposition to ICI is likely to create a selective immune memory response that is similar to that observed during infections [15]. Subsequently, the validation of this concept in prospective trials would definitely guide researchers into limiting the number of ICI cycles.

We believe that the concept of MRD using CTC represents a futuristic approach to determine the duration of treatment in patients receiving ICI. Limiting the number of treatment cycles would definitely reduce the occurrence of immune-related adverse events and financial toxicities often associated with an alteration of the patient's quality of life [16]. The use of CTC to monitor MRD as a surrogate for efficacy and duration of treatment remains quite speculative and is prone to major technical problems. The optimal actual approach requires a complete understanding of the available technologies in their biological and technical dimensions. Such information should determine the prognostic parameters that would allow a more comprehensive landmark to assess the role of CTC to improve the patient outcome. The molecular characterization and levels of CTC would identify the subgroup of patients that might benefit from a limited duration of ICI.

Financial \& competing interests disclosure The authors have no relevant affiliations or financial involvement with any organization or entity with a financial interest in or financial conflict with the subject matter or materials discussed in the manuscript. This includes employment, consultancies, honoraria, stock ownership or options, expert testimony, grants or patents received or pending, or royalties.

No writing assistance was utilized in the production of this manuscript.

\section{References}

1 Nawaz K, Webster RM. The non-small-cell lung cancer drug market. Nat. Rev. Drug Discov. 15(4), 229-230 (2016).

2 Pardoll DM. The blockade of immune checkpoints in cancer immunotherapy. Nat. Rev. Cancer 12(4), 252-264 (2012).

3 Hodi FS, O’Day SJ, McDermott DF et al. Improved survival with ipilimumab in patients with metastatic melanoma. N. Engl. J. Med. 363(8), 711-723 (2010).
4 Weber JS, D’Angelo SP, Minor D et al. Nivolumab versus chemotherapy in patients with advanced melanoma who progressed after anti-CTLA-4 treatment (CheckMate 037): a randomised, controlled, open-label, Phase III trial. Lancet Oncol. 16(4), 375-384 (2015).

5 Pantel K, Alix-Panabières C. Real-time liquid biopsy in cancer patients: fact or fiction? Cancer Res. 73(21), 6384-6388 (2013).

6 Vázquez V, Otero LL, Laurent VE, Gabri MR, Gómez DE, Alonso DF. Molecular detection of minimal residual disease in melanoma and solid tumors. Medicina (B. Aires) 69(1 Pt 2), 181-190 (2009).

7 Ignatiadis M, Reinholz M. Minimal residual disease and circulating tumor cells in breast cancer. Breast Cancer Res. 13(5), 222 (2011).

8 Bork U, Grützmann R, Rahbari NN et al. Prognostic relevance of minimal residual disease in colorectal cancer. World J. Gastroenterol. 20(30), 10296-10304 (2014). 
Tie J, Wang Y, Tomasetti C et al. Circulating tumor DNA analysis detects minimal residual disease and predicts recurrence in patients with stage II colon cancer. Sci. Transl. Med. 8(346), 346ra92 (2016).

10 Nicolazzo C, Raimondi C, Mancini M et al. Monitoring PD-L1 positive circulating tumor cells in non-small-cell lung cancer patients treated with the PD-1 inhibitor nivolumab. Sci. Rep. 6, 31726 (2016).

11 Brahmer J, Reckamp KL, Baas P et al. Nivolumab versus docetaxel in advanced squamous-cell non-small-cell lung cancer. N. Engl. J. Med. 373(2), 123-135 (2015).

12 Motzer RJ, Escudier B, McDermott DF et al. Nivolumab versus everolimus in advanced renal-cell carcinoma. $N$. Engl. J. Med. 373(19), 1803-1813 (2015).

13 Reck M, Rodríguez-Abreu D, Robinson AG et al. Pembrolizumab versus chemotherapy for PD-L1-positive non-small-cell lung cancer. N. Engl. J. Med. doi:10.1056/ NEJMoa1606774 (2016) (Epub ahead of print).
14 Lipson EJ, Sharfman WH, Drake CG et al. Durable cancer regression off-treatment and effective reinduction therapy with an anti-PD-1 antibody. Clin. Cancer Res. 19(2), 462-468 (2013).

15 Allie SR, Zhang W, Fuse S, Usherwood EJ. Programmed death 1 regulates development of central memory CD8 T cells after acute viral infection. J. Immunol. 186(11), 6280-6286 (2011).

16 Siddiqui M, Rajkumar SV. The high cost of cancer drugs and what we can do about it. Mayo Clin. Proc. 87(10), 935-943 (2012). 